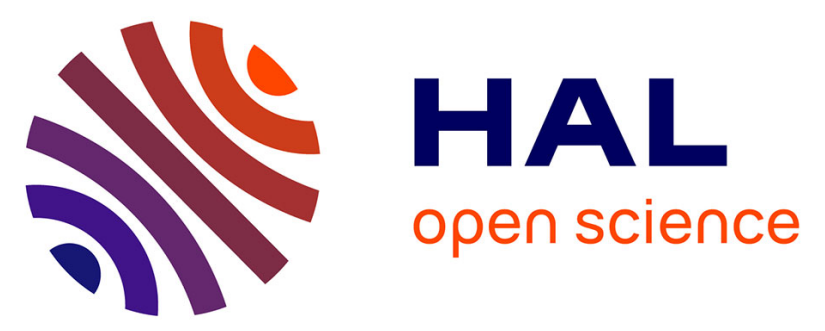

\title{
Pharmacokinetic study of intravenously administered artemisinin-loaded surface-decorated amphiphilic gamma-cyclodextrin nanoparticles
}

J.B.G. Yameogo, Roseline Mazet, Denis Wouessidjewe, Luc Choisnard, Diane Godin-Ribuot, Jean-Luc Putaux, Rasmané Semdé, Annabelle Gèze

\section{To cite this version:}

J.B.G. Yameogo, Roseline Mazet, Denis Wouessidjewe, Luc Choisnard, Diane Godin-Ribuot, et al.. Pharmacokinetic study of intravenously administered artemisinin-loaded surface-decorated amphiphilic gamma-cyclodextrin nanoparticles. Materials Science and Engineering: C, 2020, 10.1016/j.msec.2019.110281 . hal-02314466

\author{
HAL Id: hal-02314466 \\ https://hal.science/hal-02314466
}

Submitted on 10 Nov 2020

HAL is a multi-disciplinary open access archive for the deposit and dissemination of scientific research documents, whether they are published or not. The documents may come from teaching and research institutions in France or abroad, or from public or private research centers.
L'archive ouverte pluridisciplinaire HAL, est destinée au dépôt et à la diffusion de documents scientifiques de niveau recherche, publiés ou non, émanant des établissements d'enseignement et de recherche français ou étrangers, des laboratoires publics ou privés. 


\section{Pharmacokinetic study of intravenously administered artemisinin-loaded surface-decorated amphiphilic $\gamma$-cyclodextrin nanoparticles}

Josias Boumbéwendin Gérard Yaméogo ${ }^{1,3}$, Roseline Mazet ${ }^{1,2}$, Denis Wouessidjewe ${ }^{1}$, Luc Choisnard $^{1}$, Diane Godin-Ribuot ${ }^{4}$, Jean-Luc Putaux ${ }^{5}$, Rasmané Semdé ${ }^{3}$, Annabelle Gèze ${ }^{1}$

${ }^{1}$ Univ. Grenoble Alpes, CNRS, DPM, F-38000 Grenoble, France

${ }^{2}$ Pôle Pharmacie, Grenoble University Hospital, 38000 Grenoble, France

${ }^{3}$ UFR/SDS, University Ouaga I Pr Joseph Ki-Zerbo, Burkina Faso

${ }^{4}$ Laboratoire HP2/U1042 INSERM, Faculty of Pharmacy, Univ. Grenoble Alpes, France

${ }^{5}$ Univ. Grenoble Alpes, CNRS, CERMAV, F-38000 Grenoble, France

Correspondence to: Annabelle Gèze, Tel.: +33 476635301, Fax: +33 476635322, E-mail address: annabelle.geze@univ-grenoble-alpes.fr 


\begin{abstract}
Artemisinin and its derivatives are currently recommended by World Health Organization for the treatment of malaria. Severe malaria requires a parenteral administration of artemisinin-based formulations. However, the effective use of artemisinin is limited by the pharmacokinetic characteristics of the drug (low water solubility, poor bioavailability and short half-life). To overcome some of these drawbacks, artemisinin-loaded surface-decorated nanoparticles were prepared by conanoprecipitation of $\gamma$-cyclodextrin bioesterified with $\mathrm{C}_{10}$ alkyl chains and polyethylene glycol (PEG) derivatives (polysorbate 80 and DMPE-mPEG2000). Using a single dose (1.5 mg. $\mathrm{kg}^{-1}$ or $2 \mathrm{mg} \cdot \mathrm{kg}^{-1}$ ) by intravenous administration, we investigated the in vivo pharmacokinetic properties in healthy rats of two types of artemisinin-loaded nanoparticle formulations, namely, nanosphere and nanoreservoir systems versus ethanolic-aqueous solution of artemisinin as reference. Significantly enhanced pharmacokinetic parameters were obtained with artemisinin-loaded nanoparticles. In comparison to reference formulation, the geometric mean exposures in plasma $\left(\mathrm{AUC}_{0_{-\mathrm{t}}}\right)$ exhibited 2.35 and 3.26-fold increases when artemisinin was loaded in nanoreservoir and nanosphere systems, respectively. Its plasma half-life increased 4.00 and 6.25-fold and its clearance decreased up to 2.5 and 4.72-fold. Artemisinin was successfully administered intravenously by means of surface-decorated amphiphilic $\gamma$-cyclodextrin nanostructures and showed a longer elimination half-life with respect to an artemisinin solution in ethanol. Therefore, these systems are likely to provide significant advantages for the intravenous treatment of severe malaria.
\end{abstract}

\title{
Graphical abstract:
}
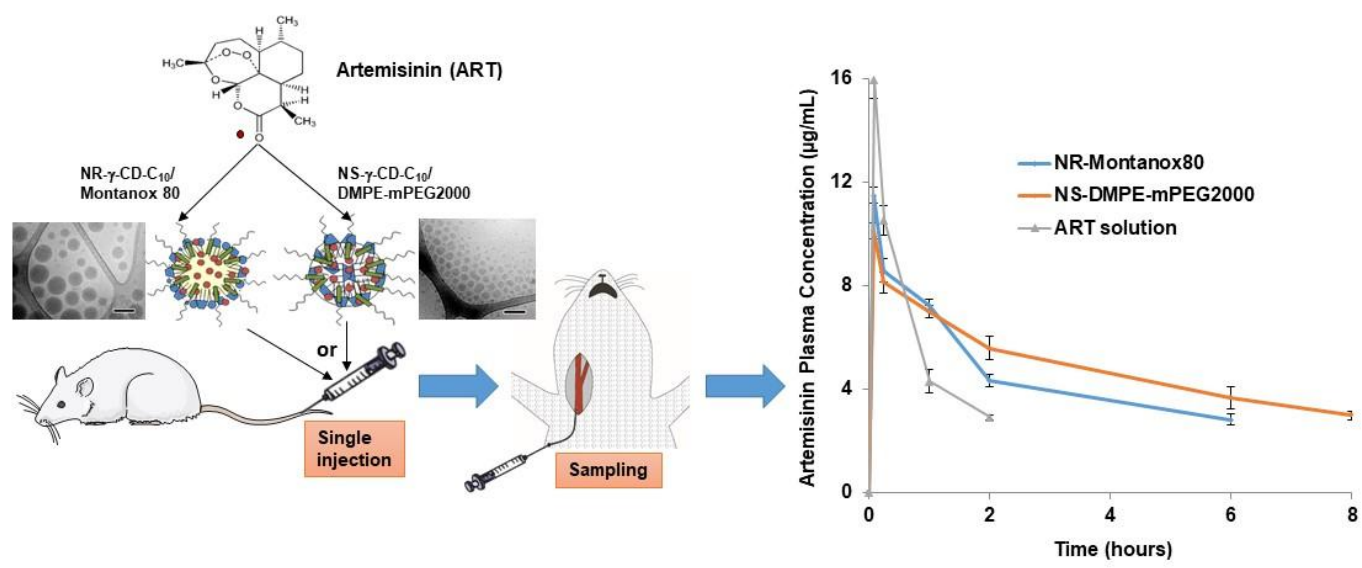

Keywords: Bioesterified $\gamma$-cyclodextrins, Artemisinin, Surface-decorated nanoparticles, Intravenous administration, Pharmacokinetic parameters, Healthy rats. 


\section{Introduction}

Malaria remains a leading cause of illness and mortality worldwide, with about 219 million cases and more than 435,000 deaths in 2017, largely in sub-Saharan Africa [1]. Artemisinin (ART) and its derivatives are powerful medicines known for their ability to swiftly reduce the number of Plasmodium parasites in the blood of patients with malaria [2]. Artemisinin, a sesquiterpene lactone endoperoxide, is a natural compound isolated from the plant Artemisia annua [3-6]. It has received considerable attention in the last decade because of its strong antimalarial activity [2,3,7]. More recently, the antiprotozoal [8,9], antibacterial [10], antiviral [11] and anticancer [12,13] properties of artemisinin have also been described.

Despite its high efficacy in the treatment of several diseases, there are important matters of concern regarding ART physical and pharmacokinetic characteristics due to its low water solubility, poor bioavailability and short half-life [3-6]. In order to improve its pharmacokinetic properties, ART is incorporated into different drug delivery systems [14]. Among the studies, the association of ART in nanoparticles or liposomes has been considered to yield improved biological outcome of ART [15-18]. Nano-encapsulation of drugs is a useful technique applied in controlled and targeted delivery. There are many literature reports on the association of ART with various nanoparticle systems (lipid, polymer, inorganic or cyclodextrin-based) which resulted in improved drug stability, prolonged drug release and enhanced bioavailability $[9,15,19]$.

Amphiphilic $\gamma$-cyclodextrin-based nanoparticles associating ART were previously investigated by our group in order to develop a parenteral formulation for the treatment of severe malaria [15]. Cyclodextrin $(\mathrm{CD})$ derivatives grafted with decanoic alkyl chains $\left(\mathrm{CD}-\mathrm{C}_{10}\right)$ were prepared by a onestep bioesterification method previously described [20-23]. Using the solvent displacement methodology, the derivatives yielded a variety of nanosphere or nanoreservoir-type systems with a size ranging from 70 to $220 \mathrm{~nm}$ [15, 20-23]. These systems could be decorated with polyethylene glycol (PEG) derivatives such as PEG1500 stearate, DMPE-mPEG2000, PEG5000 and polysorbate 80 which can reduce opsonization and phagocytosis by the macrophage cells [24,25]. PEGylated surfacedecorated $\gamma$-CD- $\mathrm{C}_{10}$ nanoparticles demonstrated non-hemolytic properties and increased blood circulation time reaching $24 \mathrm{~h}$ for nanospheres decorated with DMPE-mPEG2000 [24]. The PEGylated $\beta-C D-C_{10}$ nanospheres also presented an increased circulation time after intravenous injection, particularly when nanoparticles were surface decorated by PEG5000. These nanosystems caused neither acute nor extended acute toxicity [25] following repeated intraperitoneal injections. Both types of $\beta$ and $\gamma$-CD- $\mathrm{C}_{10}$ nanostructures were able to associate ART. The formulation parameters were optimized to reach stable and high ART dosage corresponding to drug levels of 0.3 and 1.6 $\mathrm{mg} \cdot \mathrm{mL}^{-1}$ in the colloidal suspension, for the $\gamma$-CD-C $\mathrm{C}_{10}$-based sphere and reservoir-type nanosystems, respectively. They showed a sustained in vitro release profiles of drug over 240 and 96 h, respectively. 
In vitro antimalarial activity was evaluated using the plasmodium-lactate dehydrogenase assay. ARTloaded nanoparticles inhibited the growth of cultured Plasmodium falciparum, both multi-resistant K1 and susceptible $3 \mathrm{D} 7$ strains with $\mathrm{IC}_{50}$ values of 2.8 and $7.0 \mathrm{ng} \cdot \mathrm{mL}^{-1}$ close to those of the reference ART solution [15]. The results of these previous studies highlight promising alternative formulation for injectable use of ART. The current study investigated the in vivo pharmacokinetics of ART-loaded surface-decorated amphiphilic $\gamma$-CD nanodevices after intravenous single dose administration.

\section{Materials and methods}

\subsection{Materials}

The $\gamma$-CD- $C_{10}$ fatty ester $\left(M_{w}=2714\right.$ g.mol ${ }^{-1}$, total degree of substitution $\left.=9.2\right)$ was synthesized in our laboratory by a one-step bioesterification method. Artemisinin $\left(\mathrm{C}_{15} \mathrm{H}_{22} \mathrm{O}_{5}, 98 \%, \mathrm{M}_{\mathrm{w}}=282.3{\left.\mathrm{~g} . \mathrm{mol}^{-1}\right)}^{-1}\right.$ was purchased from Carl Roth $(\mathrm{GmbH})$. Montane ${ }^{\circledR} 80$ (sorbitan mono-oleate) and Montanox ${ }^{\circledR} 80$ (polysorbate 80, ethoxylated sorbitan mono-oleate) of pharmaceutical grade were kindly provided by Seppic (Castres, France). Benzyl benzoate was supplied by Cooper (Melun, France). Dimyristoylphosphatidylethanolamine-polyethylene glycol 2000 (DMPE-mPEG2000) was obtained from Corden Pharma (Eichenweg, Switzerland). Water was freshly deionized in our laboratory.

\subsection{Preparation of colloidal suspensions}

$\gamma$-CD- $\mathrm{C}_{10}$-based nanosphere and nanoreservoir suspensions were prepared using the solvent displacement technique [15]. This method consists in injecting, under magnetic stirring (500 rpm) at $25{ }^{\circ} \mathrm{C}$, an organic solution $(10 \mathrm{~mL})$ of both amphiphilic and lipophilic constituents into an aqueous phase containing Montane ${ }^{\circledR} 80$. The organic solvent is eliminated under reduced pressure and the resulting aqueous suspension of nanoparticles were concentrated to the desired final volume in the same way [15, 20-23]. The resulting colloidal suspensions were purified by gel chromatography using Sephadex® G-75 columns (Sigma Aldrich, Sweden) in order to remove free PEGylated phospholipids in the supernatant. Prior to the administration to animals, the suspensions were isotonized with a glucose solution.

\subsection{Physicochemical characterization of the nanosystems}

The mean size, polydispersity index (PI) and zeta potential were determined after appropriate dilution using a Zetasizer (Nano Series DTS 1060, Malvern Instruments) [15,20-23]. The nanosystems were observed by transmission electron microscopy (TEM) after negative staining of the preparations with uranyl acetate or by cryo-TEM after quench-freezing of a thin film of particle suspension in liquid ethane. Both types of preparations were observed at room and low temperature, respectively, with a FEI-Philips CM200 'Cryo' microscope operating at $80 \mathrm{kV}$. The images were recorded on Kodak SO163 films [15,20-23]. The osmolarity was also measured in triplicate with an automatic microosmometer Loser Messtechnik type 15. The density of the nanoparticles in the suspension was 
determined using isopycnic centrifugation of Percoll gradients in iso-osmotic sucrose as previously described $(0.25 \mathrm{M})$ at a ratio of $1: 9(\mathrm{v} / \mathrm{v})$ [24]. The total concentration of ART recovered in the colloidal suspensions $(\mathrm{Ct})$ and the ART concentration in ultracentrifuged supernatant $(\mathrm{Cs})$ were determined, after a sample dilution with methanol, by HPLC (Varian Prostar pump; Kromasil $\mathrm{C}_{18}$ column $250 \times 4.6 \mathrm{~mm}, 5 \mu \mathrm{m}$ particle size from Supelco Inc.) coupled to evaporative light scattering detection (ELSD Sedex Model 55, Touzart \& Matignon, France) as previously described [15]. The drug recovery DR $(\%)$ and the drug association DA $(\%)$ were estimated as follows: DR $(\%)=\mathrm{Ct} / \mathrm{Ci}$ $\mathrm{x} 100$ where $\mathrm{Ci}$ is the theoretical drug concentration (initially introduced in the formulation), $\mathrm{DA}(\%)=$ $(\mathrm{Ct}-\mathrm{Cs}) / \mathrm{Ct} \times 100$. The colloidal stability of all the freshly made $\gamma-\mathrm{CD}-\mathrm{C}_{10}$ nanoparticle suspensions loaded with ART (nanospheres and nanoreservoirs) were systematically tested in electrolyte medium (0.09\% $\mathrm{NaCl}$ or PBS, $\mathrm{pH}$ 7.4). The diluted suspensions were then subjected to moderate stirring (100 rpm) at $37{ }^{\circ} \mathrm{C}$ and their particle size and polydispersity index (PI) were determined after $48 \mathrm{~h}$. The suspensions were also stored at room temperature during 24 months and their stability was evaluated in terms of appearance, particle size, polydispersity index and ART concentration.

\subsection{Animal experiments}

The animal experimental protocol was approved by the University of Grenoble Animal Ethics Committee. A total of 72 male 8 week-old Wistar rats (weight 280-340 g) obtained from Janvier Laboratory (Le Genest-Saint Isle, France) were used. The animals were housed under standardized conditions of food, temperature $\left(21 \pm 1^{\circ} \mathrm{C}\right)$ and hygrometry and exposed to a regular light/dark schedule $(12 \mathrm{~h} / 12 \mathrm{~h})$ in strict accordance with French Ministry of Agriculture regulations. All animals were kept in these conditions for one week and were divided randomly into three experimental groups, referred to as groups I, II and III.

Animals were individually weighed and anesthetized with Isoflurane $2 \%$ (Nicholas Piramal, London, England), then placed in dorsal decubitus to insert a sterile catheter into the right carotid. Catheters were filled with heparinized physiological $\mathrm{NaCl}$ solution (heparin Choay ${ }^{\circledR} 25000 \mathrm{UI} / 5 \mathrm{~mL}$, Léo Laboratory, France) to prevent their obstruction [26].

ART formulations were then administered to rats via the caudal vein (single administration at the rate $0.8 \mathrm{~mL} \cdot \mathrm{min}^{-1}$ ). Groups I and II were administrated $1.5 \mathrm{mg} \cdot \mathrm{kg}^{-1}$ of ART-loaded DMPE-mPEG2000/ $\gamma$ CD- $\mathrm{C}_{10}$ nanospheres and $2 \mathrm{mg} \cdot \mathrm{kg}^{-1}$ of ART-loaded polysorbate $80 / \gamma-\mathrm{CD}-\mathrm{C}_{10}$ nanoreservoirs, respectively. Group III was treated with $2 \mathrm{mg} \cdot \mathrm{kg}^{-1}$ of reference formulation, i.e. ART in a glucose aqueous solution containing $10 \%$ ethanol.

After injection, blood samples $(600 \mu \mathrm{L})$ were taken from the rat via the catheter placed in the right carotid. Blood samples were serially collected in heparinized tubes at each point (six rats per point): baseline ( $0 \mathrm{~min}$ ), 5 and $15 \mathrm{~min}, 1,2,4,6,8$ and $24 \mathrm{~h}$, respectively after iv administration. The sample were then immediately centrifuged at $5000 \mathrm{rpm}$ for $10 \mathrm{~min}$ at $4{ }^{\circ} \mathrm{C}$ and the supernatant plasma 
collected and stored at $-20{ }^{\circ} \mathrm{C}$ until analysis. To optimize blood sampling, the rats of each experimental group G1, G2 and G3 were distributed in three sampling series named P1, P2 and P3. The blood samples were taken at $0,5,15,60,120$ min with the rats of the P1 sampling series, those at times 6 and $8 \mathrm{~h}$ with the P2 series and $24 \mathrm{~h}$ with the P3 series. Thus, the total amount of blood taken from each rat did not exceed $3,000 \mu \mathrm{L}$ (i.e. $1 / 7^{\text {th }}$ of the total blood volume of rats).

To minimize the risks of hypovolemia, an equivalent volume $(600 \mu \mathrm{L})$ of heparinized physiological solution $(0.09 \% \mathrm{NaCl})$ was administered after each blood sampling. The hematocrit of each sample was also checked to control the absence of hemodilution.

\subsection{Quantification of ART in the plasma}

ART was quantified in the plasma by using a HPLC-ELSD method after preliminary extraction following a procedure described by Ashton et al. with minor modifications [27-29]. 600 $\mu \mathrm{L}$ of phosphate buffer $(0.3 \mathrm{M}, \mathrm{pH} 3.5)$ and $60 \mu \mathrm{L}$ of hydrogen peroxide $\left(\mathrm{H}_{2} \mathrm{O}_{2}, 30 \%\right)$ were added to $300 \mu \mathrm{L}$ of each plasma sample to precipitate proteins. The resulting mixture was plunged into ice-bath in which it was submitted to magnetic stirring for $15 \mathrm{~min}$. Exactly $2.4 \mathrm{~mL}$ of hexane $/ n$-butanol $(94: 6 \mathrm{v} / \mathrm{v})$ solution was then added to the solution to extract ART. After centrifugation at $5000 \mathrm{rpm}$ for $10 \mathrm{~min}$ at $4{ }^{\circ} \mathrm{C}$, the organic phase $(2 \mathrm{~mL})$ was transferred into another tube and dried with nitrogen gas. The residue obtained was reconstituted in $200 \mu \mathrm{L}$ of methanol (HPLC mobile phase), vortexed for $2 \mathrm{~min}$ and filtrated by using nylon filter. Finally, an aliquot of $5 \mu \mathrm{L}$ for each preparation was injected into the HPLC-ELSD system for ART quantification analysis.

\subsection{Data analysis}

The pharmacokinetic parameters of ART after intravenous administration were determined from the obtained plasma concentration-time data by using non-compartmental pharmacokinetic analyses by Macro-Excel software. The trapezoidal method was used to calculate the area under the curve (AUC) of plasma concentration as a function of time and the total area under the first moment curve up to last time point $\left(\mathrm{AUC}_{0 \rightarrow t}\right)$. The area under the curve of the plasma concentrations to the first moment up to infinite time $\left(\mathrm{AUC}_{0-\infty}\right)$ is obtained by extrapolation on the lower limits. Delta AUC represents the value of the extrapolation. The elimination rate constant $\left(\mathrm{k}_{\mathrm{e}}\right)$ was calculated by the semi-logarithmic regression analysis from the slope of the line. The half-life $\left(\mathrm{t}_{1 / 2}\right)$ of the drug was obtained by dividing 0.693 by $\mathrm{k}_{\mathrm{e}}$. The total clearance was calculated by dividing the dose administered by $\mathrm{AUC}_{0-\infty}$. The volume of distribution was calculated using the equation $\mathrm{Vd}=\mathrm{CL} / \mathrm{k}$. The maximum plasma concentration $\left(C_{\max }\right)$ and the time to reach this maximum $\left(T_{\max }\right)$ were directly determined. The plasma concentration values as a percentage of the injected dose were also calculated. All data are expressed as mean value \pm standard deviation (SD). Statistical analyses were performed using one-way ANOVA test, and the level of significance was set at $5 \%$. 


\section{Results and discussion}

\subsection{Physicochemical characterization of ART-loaded surface-decorated $\gamma-C D$ - $C_{10}$ nanoparticles}

ART was loaded in PEGylated surface-decorated $\gamma$-CD-C $\mathrm{C}_{10}$ nanoparticles via a co-nanoprecipitation

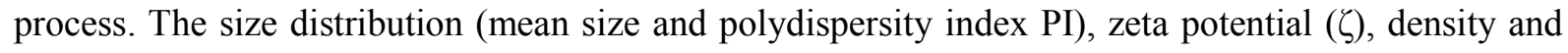
drug content of nanospheres and nanoreservoirs are reported in Table 1. The colloidal systems were characterized by a mean size of $90 \mathrm{~nm}$ for nanospheres and $190 \mathrm{~nm}$ for nanoreservoir systems, both suitable for intravenous injection. The nanoparticle density was in the range of blood density. Both nanosuspensions had sufficient and stable charge loading in ART corresponding to drug levels reaching 0.48 and $1.65 \mathrm{mg} \cdot \mathrm{mL}^{-1}$ for nanospheres and nanoreservoirs, respectively. These concentrations correspond to an increase of ART dose dissolved in aqueous medium by a factor of 7.5 and 25.8, respectively. The ART amount in the colloidal suspensions correspond to drug recovery (DR) close to $95 \%$ in both systems and drug association (DA) of $88 \%$ for nanospheres and $96 \%$ for nanoreservoir systems. In the case of nanoreservoirs, ART is mainly located in the lipophilic inner core of nanoparticles made of benzyl benzoate due to the high solubility of the drug in this medium (83 mg.mL ${ }^{-1}$ at $25{ }^{\circ} \mathrm{C}$ ) as compared to the low ART hydrosolubility $\left(0.06 \mathrm{mg} \cdot \mathrm{mL}-1\right.$ at $25^{\circ} \mathrm{C}$ ) [14]. In the case of nanospheres, it was assumed that there are several sites for the drug location within the nanostructures. It is also quite likely that part of the drug interacts with the cavity of $\gamma$-CD-C $\mathrm{C}_{10}$. It is well known that the solvent displacement technique allows to associate significant amounts of drug with a large part that is adsorbed on the surface. No ART desorption was observed with time indicating that ART does not form stacking layers at the nanosphere surface. The formulation conditions were previously optimized $[14,23]$ in order to avoid this phenomenon. Here again, the low ART water solubility is in favour of an ART loading in $\gamma-\mathrm{CD}-\mathrm{C}_{10}$ based nanoparticles. The physicochemical characteristics of ART-loaded surface-decorated nanoparticles were in good agreement with our previous studies [24] which showed that the introduction of the PEGylated phospholipid in the formulation induced a slight increase in average diameter and a stronger negative zeta potential values (about -20 and $-30 \mathrm{mV}$ for DMPE-PEG2000-coated $\gamma$-CD- $\mathrm{C}_{10}$ nanospheres and nanoreservoirs, respectively). The slight increase in particle size was in favor of the presence of the hydrophilic PEG corona and the apparent electric negative charge may be attributed to the presence of phosphatidyl negative charge of the phospholipid moiety. 


\section{Table 1}

Physicochemical characteristics of ART-loaded surface-decorated $\gamma$-CD- $\mathrm{C}_{10}$ nanospheres and nanoreservoirs (mean $\pm \mathrm{SD}, \mathrm{n}=3$ ).

\begin{tabular}{|c|c|c|c|c|c|c|c|}
\hline Formulation & $\begin{array}{c}\text { Mean } \\
\text { size }(\mathbf{n m})\end{array}$ & PI & $\begin{array}{c}\zeta \\
(\mathrm{mV} \pm \mathrm{SD})\end{array}$ & $\begin{array}{l}\text { ART content } \\
\left(\mathbf{m g} \cdot \mathrm{mL}^{-1}\right)\end{array}$ & DR (\%) & DA (\%) & $\begin{array}{c}\text { Density at } \\
20^{\circ} \mathrm{C}\left(\mathrm{g} \mathrm{cm}^{-3}\right)\end{array}$ \\
\hline $\begin{array}{l}\text { ART-loaded DMPE- } \\
\text { mPEG2000/ } \gamma-C D-C_{10} \\
\text { nanospheres }\end{array}$ & 92 & 0.15 & $-22 \pm 1$ & 0.48 & 95.05 & 88.00 & 1.095 \\
\hline $\begin{array}{l}\text { ART-loaded polysorbate } \\
80 / \gamma-C D-C_{10} \\
\text { nanoreservoirs }\end{array}$ & 188 & 0.06 & $-29 \pm 2$ & 1.65 & 95.52 & 96.33 & 1.069 \\
\hline
\end{tabular}
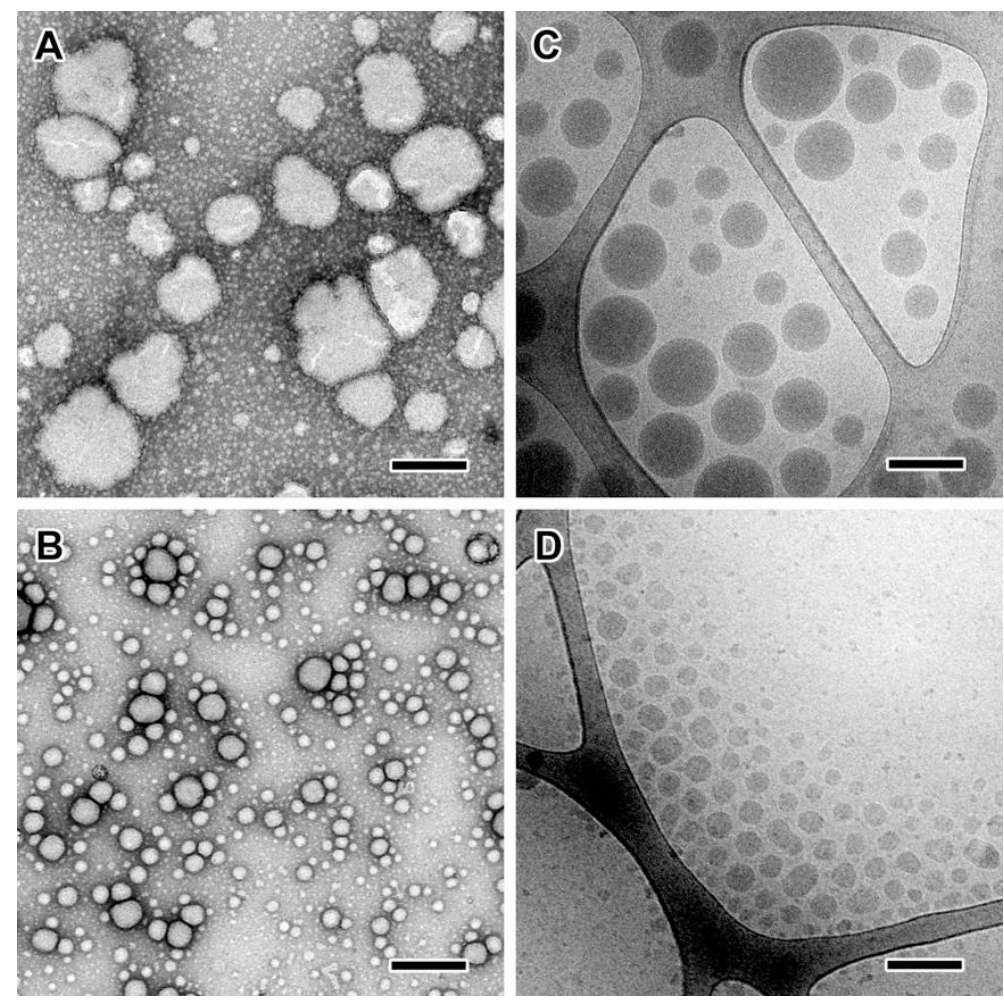

Figure 1. TEM and cryo-TEM images of negatively stained (A,B) and ice-embedded (C,D) ART-loaded polysorbate $80 / \gamma-\mathrm{CD}-\mathrm{C}_{10}$ nanoreservoirs $(\mathrm{A}, \mathrm{C})$ and ART-loaded DMPE-mPEG2000/ $\gamma-\mathrm{CD}-\mathrm{C}_{10}$ nanospheres (B,D). Bars: $200 \mathrm{~nm}$.

Figure 1 shows TEM and cryo-TEM images of negatively stained and ice-embedded nanoparticle preparations, respectively. As previously reported [23], the shape of dried nanoreservoirs is not well defined due to the deformation and flattening of the liquid-like particles on the carbon film (Fig. 1A). The ice-embedded nanoreservoirs appear as electron-dense spherical particles with a diameter ranging from 70 to $200 \mathrm{~nm}$ (Fig. 1C), in good agreement with the DLS data. In comparison, the $\gamma$-CD-C 10 nanospheres are slightly facetted solid particles that did not deform upon drying since their size 
distribution (20-100 $\mathrm{nm}$ in diameter) is similar in images of dry (Fig. 1B) and ice-embedded (Fig. 1D) specimens.

As seen in Figure 2, ART-loaded nanospheres and nanoreservoirs stored at ambient temperature presented a good physical stability at 24 months. The amount of entrapped drug remained stable as well during this period $\left(0.48\right.$ and $1.64 \mathrm{mg} \mathrm{mL}^{-1}$ for ART loaded nanospheres and nanoreservoirs respectively). Moreover, after dilution in electrolyte medium, the nanoparticle size and polydispersity of both nanosuspensions were unchanged after $48 \mathrm{~h}$, indicating the absence of aggregation over time.

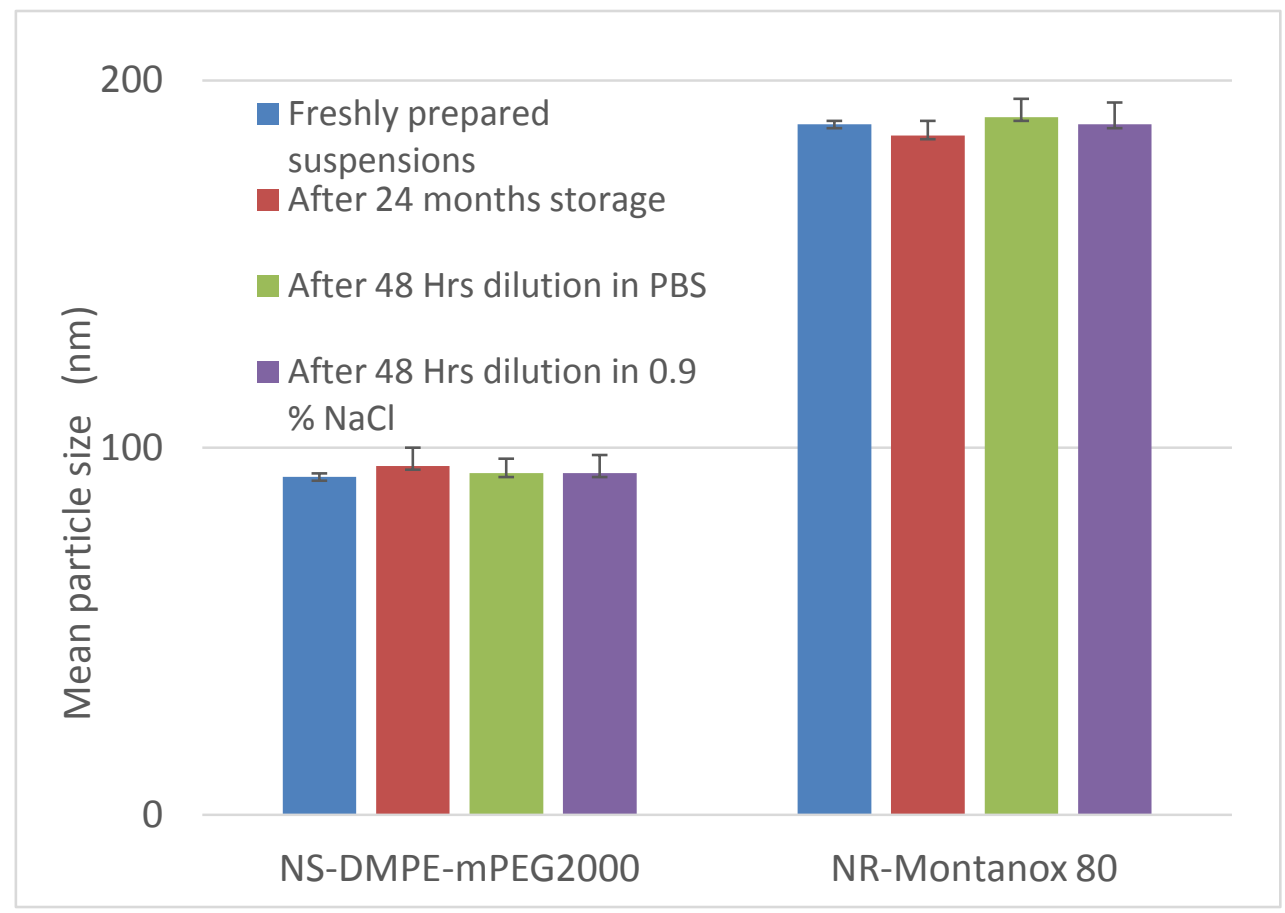

Figure 2. Mean particle sizes of ART-loaded polysorbate $80 / \gamma-\mathrm{CD}-\mathrm{C}_{10}$ nanoreservoirs and ART-loaded DMPEmPEG2000/ $\gamma-\mathrm{CD}-\mathrm{C}_{10}$ nanospheres after 24 months storage at ambient temperature and after $48 \mathrm{~h}$ in $0.09 \% \mathrm{NaCl}$ or PBS pH 7.4 solutions (mean $\pm \mathrm{SD}, \mathrm{n}=3$ ).

\subsection{In vivo pharmacokinetic study of ART-loaded surface decorated $\gamma-C D$ - $C_{10}$ nanostructures}

The pharmacokinetic study has been conducted in order to find out whether there was any enhancement in the bioavailability of ART from ART-loaded surface-decorated $\gamma$-CD-C $\mathrm{C}_{10}$ nanoparticles. The reference solution was injected at a dose of $2 \mathrm{mg} \cdot \mathrm{kg}^{-1}$. In the case of nanoreservoirs, the animals received a $400 \mu \mathrm{L}$ injection corresponding to a similar dose of $2 \mathrm{mg} \cdot \mathrm{kg}^{-1}$. However, in the case of nanosphere suspensions, the ART dose injected in animals was slightly lower. Indeed, the maximum injectable volume in rats $(800 \mu \mathrm{L})$ made it possible to reach a maximum dose of $1.5 \mathrm{mg} . \mathrm{kg}^{-}$ 1. The amount of blood sample taken from the rat (about $14 \%$ of the total blood volume) cannot affected the pharmacokinetic experiment. Indeed, it is recognized that no more than $20 \%$ of the total blood volume should be taken at one time or within 14 days in animal. In addition, the values of the 
hematocrit determined at each sample give good indications concerning the physiological state of the animal.

The pharmacokinetic profiles of ART were determined using the rat plasma concentration-time curve obtained after intravenous administration of ART loaded nanospheres and nanoreservoirs. The mean plasma concentration-time profiles are presented in Figure 2 and their corresponding pharmacokinetic parameters are shown in Table 2.

From Figure 3, the ART reference solution displayed higher initial mean plasma concentration with an observed $\mathrm{C}_{\max }$ of $15.92 \mu \mathrm{g} \cdot \mathrm{mL}^{-1}$ after 5 min of administration of a dose of $2 \mathrm{mg} . \mathrm{kg}^{-1}$ against 11.50 $\mu \mathrm{g} \cdot \mathrm{mL}^{-1}$ and $10.12 \mu \mathrm{g} \cdot \mathrm{mL}^{-1}$ after $5 \mathrm{~min}$ administration of $2 \mathrm{mg} \cdot \mathrm{kg}^{-1}$ of ART-loaded nanoreservoirs and $1.5 \mathrm{mg} \cdot \mathrm{kg}^{-1}$ of ART-loaded nanosphere suspensions, respectively. The initial plasma concentration level of ART-loaded nanoparticles corresponds to approximately 30 and $45 \%$ of the injected dose, respectively, for nanoreservoir and nanosphere suspensions. These levels were correlated with the previous in vitro release data which showed a burst effect corresponding to 32 and $50 \%$ of dissolved drug in the tested release medium after $30 \mathrm{~min}$ for nanoreservoir and nanosphere suspensions respectively [15].

The concentration of ART in all formulations showed an expected declining trend. The ART from the reference solution was rapidly eliminated from the plasma and was no longer detectable after $2 \mathrm{~h}$ (halflife $=0.81 \pm 0.1 \mathrm{~h})$. It can be mentioned that a plasma half-life of the same order $(0.85-1.17 \mathrm{~h}$ and 0.5 h) was obtained by Dai et al. [30] and Ashton et al. [31] after an intravenous administration of a methanol solution (10 mg. $\left.\mathrm{kg}^{-1}\right)$ and aqueous emulsion $\left(20 \mathrm{mg} \cdot \mathrm{kg}^{-1}\right)$ of ART to rats. The plasma halflife of ART after oral administration was also in the same range (0.76-2.21 h) [28, 30].
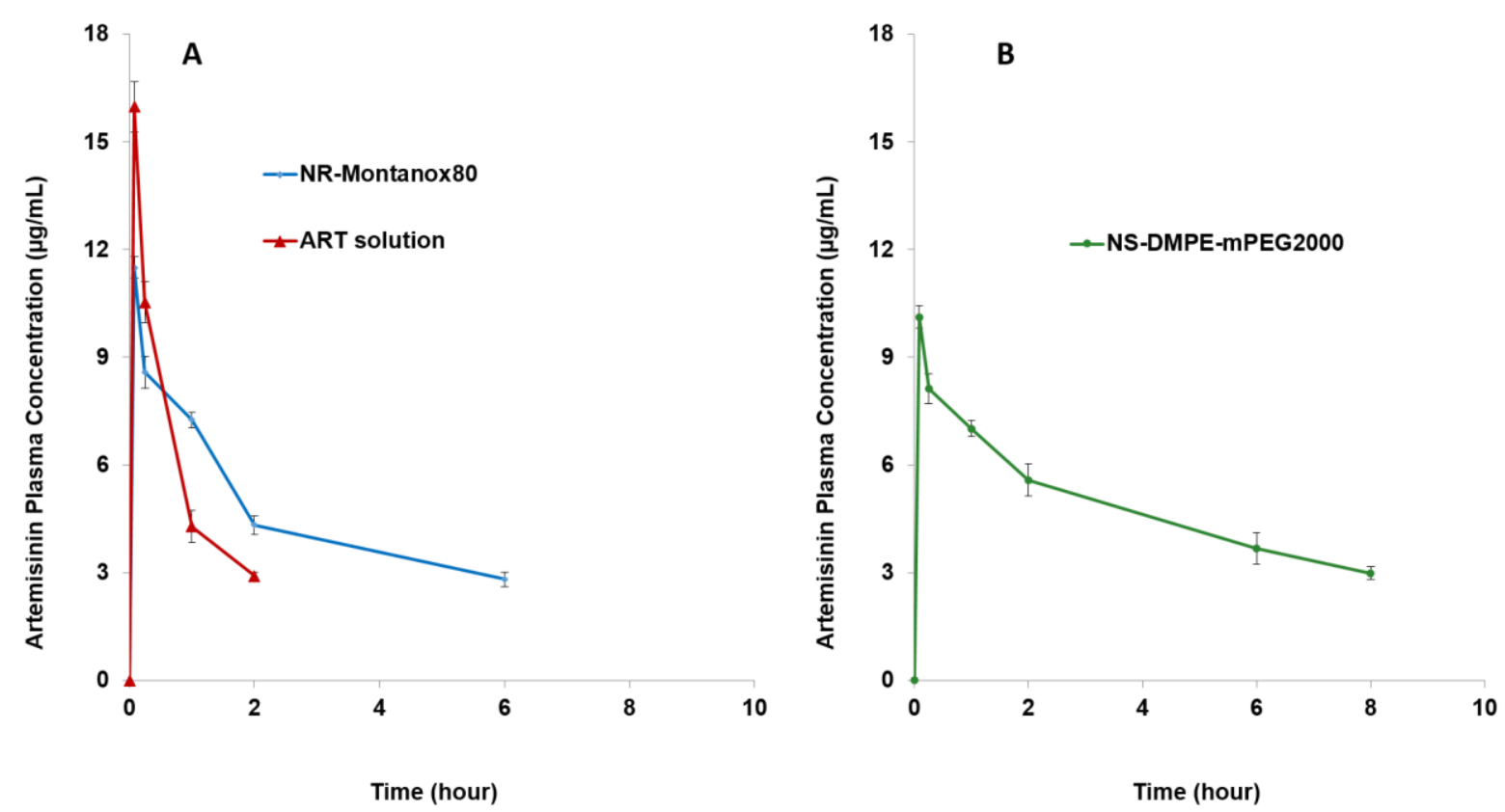
Figure 3. Mean plasma concentration-time profiles of ART after single intravenous dose $(n=8)$ of $($ A) 2 mg. $\mathrm{kg}^{-1}$ of ART-loaded polysorbate $80 / \gamma-\mathrm{CD}-\mathrm{C}_{10}$ nanoreservoirs and ART solution in $10 \%$ ethanol; (B) 1.5 mg.kg ${ }^{-1}$ of ART-loaded DMPE-mPEG2000/ $\gamma-\mathrm{CD}-\mathrm{C}_{10}$ nanospheres (mean $\pm \mathrm{SD}, \mathrm{n}=3$ ).

ART-loaded nanosystems displayed a marked improvement in the circulation behavior over time as compared to the ART solution. The mean plasma concentrations of the ART in nanosuspensions gradually decreased to a level corresponding to the limit of quantification of the employed HPLC method $\left(2.8 \mu \mathrm{g} \cdot \mathrm{mL}^{-1}\right)$, after $6 \mathrm{~h}$ (nanoreservoirs) and $8 \mathrm{~h}$ (nanospheres). The mean concentrations detected in this last point correspond to approximately 10 and $13 \%$ of the administered dose, respectively.

The first pharmacokinetic goal in malaria treatment is to achieve a fast onset of action with a sufficient concentration of antimalarial at the shortest possible time to clear parasite load effectively. The $\mathrm{IC}_{50}$ value of $7.0 \mathrm{ng} \cdot \mathrm{mL}^{-1}$ was considered for antimalarial action of ART [15]. The initial plasma concentrations of $\mathrm{ART}$ obtained were above the $\mathrm{IC}_{50}$ value. Despite the decrease over time, plasma concentrations of ART (10 to $3 \mu \mathrm{g} \cdot \mathrm{mL}^{-1}$ ) also remained above the $\mathrm{IC}_{50}$ value, 6 and $8 \mathrm{~h}$ after intravenous administration of $2 \mathrm{mg} \cdot \mathrm{kg}^{-1}$ of ART-loaded nanoreservoirs and $1.5 \mathrm{mg} . \mathrm{kg}^{-1}$ of ART-loaded nanospheres, respectively.

The plasma concentration-time profiles obtained with ART-loaded nanostructures were biphasic with a rapid distribution and a slowed elimination phases in comparison to ART reference solution. The plasma half-life of ART from nanoreservoirs and nanospheres formulations were $3.23 \pm 0.16$ and $5.03 \pm 0.24 \mathrm{~h}$, respectively. In comparison with ART reference solution, the plasma half-life values increased by 4.00 and 6.25 -fold for $\gamma$-CD- $\mathrm{C}_{10}$ nanoreservoirs and nanospheres, respectively. This clearly indicated that ART-loaded surface-decorated amphiphilic $\gamma$-CD- $\mathrm{C}_{10}$ nanoparticles remained in the blood stream as previously observed [23] thus allowing nanostructures to release ART in the blood in a sustained manner. The mean plasma half-life of ART from $\gamma$-CD-C $\mathrm{C}_{10} / \mathrm{DMPE}-\mathrm{mPEG} 2000$ nanospheres was 1.56 -fold higher than that of $\gamma$-CD- $\mathrm{C}_{10}$ polysorbate 80 nanoreservoirs, despite the greater $\mathrm{C}_{\max }$ obtained with nanoreservoir suspensions.

These results make it possible to meet the main objective of associating ART to nanodevices which is to improve drug plasma half-life and its bioavailability. Isacchi et al. [32] observed half-life times of 0.38, 0.67 and $2.02 \mathrm{~h}$ after intra-peritoneal administration in mice of ART $\left(10 \mathrm{mg} \cdot \mathrm{kg}^{-1}\right)$ solubilized in DMSO (10 \% v/v) or associated to conventional or PEGylated liposomes, respectively. However, the half-life values obtained with PEGylated liposomal formulations (2.02 h) [32] were below that obtained with both $\gamma$-CD- $\mathrm{C}_{10}$-based nanoparticles. Similar bioavailability improvement conclusions can be drawn from Table 2, that shows the pharmacokinetic parameters associated to $\gamma$-CD$\mathrm{C}_{10} / \mathrm{DMPE}-\mathrm{mPEG} 2000$ nanospheres, $\gamma$-CD- $\mathrm{C}_{10}$ /polysorbate 80 nanoreservoirs and reference $\mathrm{ART}$ solution. 
The plasma clearance of ART from the reference solution, ART-loaded $\gamma$-CD- $\mathrm{C}_{10} /$ polysorbate 80 nanoreservoirs and ART-loaded $\gamma$-CD- $\mathrm{C}_{10} / \mathrm{DMPE}-\mathrm{mPEG} 2000$ nanospheres were respectively $34.88 \pm 1.33$ L.h ${ }^{-1}, 13.79 \pm 0.77$ L.h ${ }^{-1}$ and $7.40 \pm 0.37$ L.h ${ }^{-1}$ showing that surface-decorated $\gamma$-CD-C ${ }_{10}$ nanostructures were able to increase the residence time of drug in the plasma resulting in slow elimination from the blood stream. The ART clearance in both nanoparticle formulations decreased up to 2.5 and 4.72-fold, respectively. As can be seen, the ART clearance is significantly $(\mathrm{p}<0.01)$ reduced with the nanospheres compared to nanoreservoirs suggesting a higher hepatic extraction of nanoreservoirs. This result is consistent with the biodistribution data which showed a better vascular remanence with $\gamma$-CD-C 10 DMPE-mPEG2000 nanospheres [24].

Table 2.

Pharmacokinetic parameters of artemisinin after intravenous administration of ART-loaded polysorbate 80/ $\gamma$ CD-C 10 nanoreservoirs $\left(2 \mathrm{mg} . \mathrm{kg}^{-1}\right)$, ART-loaded DMPE-mPEG2000/ $\gamma-C D-C_{10}$ nanospheres $\left(1.5 \mathrm{mg} \cdot \mathrm{kg}^{-1}\right)$ and ART solution in $10 \%$ ethanol.

\begin{tabular}{|c|c|c|c|}
\hline $\begin{array}{l}\text { Pharmacokinetic } \\
\text { parameters }\end{array}$ & $\begin{array}{c}\text { ART (in 10\% ethanol } \\
\text { solution) }\end{array}$ & $\begin{array}{c}\text { ART-loaded polysorbate } \\
\text { 80/ } \gamma \text {-CD-C } \text { C }_{10} \text { nanoreservoirs }\end{array}$ & $\begin{array}{c}\text { ART-loaded DMPE-mPEG2000/ } \gamma \text { - } \\
\text { CD-C } \text { C }_{10} \text { nanospheres }\end{array}$ \\
\hline $\mathrm{AUC}_{0-\mathrm{t}}\left(\mu \mathrm{g} \cdot \mathrm{h} \cdot \mathrm{mL}^{-1}\right)$ & $12.04 \pm 0.49$ & $28.19 \pm 1.00$ & $39.14 \pm 2.31$ \\
\hline $\mathrm{AUC}_{0 \text {-inf }}\left(\mu\right.$ g.h. $\left.\mathrm{mL}^{-1}\right)$ & $15.43 \pm 0.52$ & $41.34 \pm 2.31$ & $60.83 \pm 3.11$ \\
\hline delta AUC & $3.39 \pm 0.14$ & $13.15 \pm 1.53$ & $21.70 \pm 1.93$ \\
\hline$\%$ de delta AUC & $28.18 \pm 1.49$ & $46.66 \pm 4.48$ & $55.44 \pm 5.67$ \\
\hline $\mathrm{t}_{1 / 2}(\mathrm{~h})$ & $0.81 \pm 0.10$ & $3.23 \pm 0.16$ & $5.03 \pm 0.24$ \\
\hline $\mathrm{k}_{\mathrm{e}}$ & $0.89 \pm 0.01$ & $0.21 \pm 0.01$ & $0.14 \pm 0.01$ \\
\hline $\mathrm{C}_{\max }\left(\mu \mathrm{g} \cdot \mathrm{mL}^{-1}\right)$ & $15.92 \pm 0.71$ & $11.50 \pm 0.31$ & $10.12 \pm 0.31$ \\
\hline CL $\left(L \cdot h^{-1}\right)$ & $34.88 \pm 1.33$ & $13.79 \pm 0.77$ & $7.40 \pm 0.37$ \\
\hline $\mathrm{Vd}(\mathrm{L})$ & $39.16 \pm 1.57$ & $64.31 \pm 1.65$ & $53.68 \pm 1.26$ \\
\hline
\end{tabular}

The data summarized in Table 2 show that pharmacokinetic parameters such as $\mathrm{t}_{1 / 2}$, AUC, CL, etc., are significantly different between $\gamma$-CD- $\mathrm{C}_{10}$ nanoparticle suspensions and the ART reference solution at $\mathrm{p}<0.05$. Significantly higher geometric mean plasma exposures $\left(\mathrm{AUC}_{0-\mathrm{t}}\right)(\mathrm{p}<0.01)$ compared to reference formulation were observed with the ART-loaded surface decorated $\gamma$-CD- $\mathrm{C}_{10}$ nanoreservoir and nanosphere particles resulting in a 2.35 and 3.26-fold increase, respectively. All pharmacokinetic parameters evidenced that both nanostructures of $\gamma-\mathrm{CD}-\mathrm{C}_{10}$ protect ART from rapid elimination, with respect to longer mean residence time, decreased $\mathrm{Vd}$ and $\mathrm{CL}$ values in comparison to the ART reference solution. The pharmacokinetic parameters illustrate the effectiveness of surface-decorated $\gamma$ $\mathrm{CD}_{-} \mathrm{C}_{10}$ nanoparticles as a potential injectable delivery system for ART. The enhanced pharmacokinetic parameters $\left(\mathrm{Cmax}\right.$ and $\mathrm{t}_{1 / 2}$ ) similar to those observed with ART-loaded surface decorated $\gamma-\mathrm{CD}-\mathrm{C}_{10}$ nanoreservoirs were obtained by Manjili et al. [33] after a single dose intravenous injection in rats of $25 \mathrm{mg} \cdot \mathrm{kg}^{-1}$ of ART-loaded poly ( $\varepsilon$-caprolactone)-poly (ethylene glycol)-poly ( $\varepsilon$ caprolactone, PCL-PEG-PCL) micelles. However, in the case of ART-loaded nanospheres, $\mathrm{t}_{1 / 2}$ reached $5 \mathrm{~h}$, which was slightly higher than the value obtained with copolymer micelles described by Manjili 
et al. [33]. Moreover, the ART mean plasma exposures $\left(\mathrm{AUC}_{0-\mathrm{t}}\right)$ are significantly higher with both nanoreservoirs and nanospheres systems as compared to ART-loaded PCL-PEG-PCL micelles, despite an ART administered dose 10 fold higher than the one we used. This suggested a gradual drug release in the early hours from injected ART loaded $\gamma$-CD-C $\mathrm{C}_{10}$ nanocarriers (either nanoreservoirs or nanospheres) that allows effective drug plasma concentrations to be maintained thanks to the persistent nature of nanoparticles in the blood [24]. These results indicate that there is little loss of ART after intravenous injection aptly linked to their association with $\gamma-\mathrm{CD}-\mathrm{C}_{10}$ nanocarriers, considering that a drug fraction remains associated to the nanocarriers. It should be noted that ARTloaded DMPE-mPEG2000/ $\gamma$-CD-C 10 nanospheres displayed better improved pharmacokinetic profiles compared to polysorbate $80 / \gamma-\mathrm{CD}-\mathrm{C}_{10}$ nanoreservoirs. These results may be partly explained by the release kinetics of ART in vitro [15] as well as the biodistribution features specific to each nanosystem [24]. The overall behaviour of nanoparticles is rather conducive to effective management of severe malaria.

Besides the pharmacokinetic study, the hematocrit levels measured on each blood sample remained constant, indicating that the intravenous administration of the drug-loaded nanodevices did not cause any lysis of the red blood cells. Indeed, the hematocrit rates measured in the blood samples collected before and after each injection were constant, around 34-35\%. These additional data indicate that the injection of nanosuspensions was relatively well tolerated taking into account our experimental conditions.

\section{Conclusion}

The present comparative pharmacokinetic study in healthy rats shows that ART-loaded surfacedecorated $\gamma$-CD-C $\mathrm{C}_{10}$ nanoparticles suspensions displayed improved pharmacokinetic properties after single intravenous administration. The results showed that $\gamma-\mathrm{CD}-\mathrm{C}_{10}$ nanostructures are able to maintain ART plasma concentrations above the therapeutically relevant concentration for a minimum period of 6 and $8 \mathrm{~h}$, for ART-loaded polysorbate 80/ $\gamma-\mathrm{CD}-\mathrm{C}_{10}$ nanoreservoirs and ART-loaded DMPEmPEG2000/ $\gamma-C D-C_{10}$ nanospheres, respectively. However, the nanosphere-type system exhibited improved pharmacokinetic characteristics compared to those of the reservoir-type. This study is in agreement with our previous observations and highlights the potential of surface-decorated $\gamma$-CD-C $\mathrm{C}_{10}$ nanoparticles as intravenous ART delivery system in the perspective of therapeutic management of severe malaria. Further in vivo explorative studies will establish a good correlation and support the pharmacokinetics data.

\section{Acknowledgments}


The authors thank Christine Brunet-Manquat, Delphine Levilly (DPM), and Emeline Lemarie (HP2) for their technical assistance, as well as the NanoBio-ICMG Platform (FR 2607, Grenoble) for granting access to the Electron Microscopy facility. We also thank the French Embassy in Burkina Faso for the financial support of J.B.G. Yaméogo. This work has also been partially supported by grants from Agence Nationale de la Recherche (grant \#ANR-11-BS10-0007) and LabEx ARCANE (ANR-11-LABX-0003-01).

\section{References}

[1] World Health Organization Geneva, World Malaria Report 2018.

[2] L. Cui, X.Z. Su, Discovery, mechanisms of action and combination therapy of artemisinin, Exp. Rev. Anti. Infect. Ther. 7 (2009) 999-1013.

[3] N.J. White, Qinghaosu (artemisinin): the price of success, Science 320 (2008) 330-334.

[4] G. Zongru, Artemisinin anti-malarial drugs in China, Acta Pharm. Sin. B (2016) 115-124.

[5] C.X Liu, Discovery and development of artemisinin and related compounds, Chinese Herbal Medicines, 9(2) (2017) 101-114.

[6] H.A. Karunajeewa, Artemisinins: artemisinin, dihydroartemisinin, artemether and artesunate, Milestones in Drug Therapy 41 (2012) 157-190.

[7] W.E. Ho, H.Y. Peh, T.K. Chan, W.S Wong, Artemisinins: pharmacological actions beyond anti-malarial, Pharmacol. Ther. 142 (2014) 126-139.

[8] M.E.M. Saeed, S. Krishna, H.J. Greten, P.G. Kremsner, T. Efferth, Antischistosomal activity of artemisinin derivatives in vivo and in patients, Pharmacol. Res. 110 (2016) 216-226.

[9] M.Y. Want, M. Islamuddin, G. Chouhan, H.A. Ozbak, H.A. Hemeg, A.K. Dasgupta, A.P. Chattopadhyay, F. Afrin, Therapeutic efficacy of artemisinin-loaded nanoparticles in experimental visceral leishmaniasis. Colloid Surf. B 130 (2015) 215-221.

[10]L. Lin, X. Mao, Y. Sun, H. Cui, Antibacterial mechanism of artemisinin / beta-cyclodextrins against methicillin-resistant Staphylococcus aureus (MRSA), Microb. Pathog. 118 (2018) 66-73.

[11] T. Efferth, Beyond malaria: The inhibition of viruses by artemisinin-type compounds, Biotechnol. Adv. 36 (2018) $1730-1737$.

[12] S. Slezakova, J. Ruda-Kucerova, Anticancer activity of artemisinin and its derivatives, Anticancer Res. 37(11) (2017) 5995-6003.

[13]H. Nosrati, P. Barzegari, H. Danafar H.K. Manjili, Biotin-functionalized copolymeric PEG-PCL micelles for in vivo tumour-targeted delivery of artemisinin, Artif. Cells Nanomed. Biotechnol. 47 (1) (2019) 104114

[14] A. Ba, Design of drug delivery systems containing artemisinin and its derivatives, Molecules 22 (2) (2017) 323-342.

[15] J.B. Yaméogo, A. Gèze, L. Choisnard, J.-L. Putaux, A. Gansané, S.B. Sirima, R. Semdé, D. Wouessidjewe, Self-assembled biotransesterified cyclodextrins as Artemisinin nanocarriers. I: Formulation, lyoavailability and in vitro antimalarial activity assessment, Eur. J. Pharm. Biopharm. 80 (2012) 508-517.

[16] N. Ibrahim, H. Ibrahim, A.M. Sabater, D. Mazier, A. Valentin, F. Nepveu, Artemisinin nanoformulation suitable for intravenous injection: Preparation, characterization and antimalarial activities, Int. J. Pharm. 495 (2015) 671-679.

[17]B. Isacchi, M.C. Bergonzi, M. Grazioso, C. Righeschi, A. Pietretti, C. Severini, A.R. Bilia, Artemisinin and artemisinin plus curcumin liposomal formulations: Enhanced antimalarial efficacy against Plasmodium berghei-infected mice, Eur. J. Pharm. Biopharm. 80 (2012) 528-534. 
[18]D. Neda, N. Dariush, C. Mohsen, E.S. Hassan, M.S. Mohammadreza, F. Ali, A. Akbarzadeh, Effect of artemisinin liposome and artemisinin liposome polyethylene glycol on MCF-7 cell line. Int. J. Life Sci. Biotechnol. Pharm. Res. 2 (2013) 349-355.

[19] J. Chen, Z. Guo, H.B. Wang, J.J. Zhou, W.J. Zhang, Q.W. Chen, Multifunctional mesoporous nanoparticles as $\mathrm{pH}$-responsive $\mathrm{Fe}^{2+}$ reservoirs and artemisinin vehicles for synergistic inhibition of tumor growth, Biomaterials 35 (2014) 6498-6507.

[20]L. Choisnard, A. Gèze, C. Vanharverbeke, J.B.G. Yaméogo, J.-L Putaux, B. Brasme, L. Julien, S. Boulanger, C. Elfakir, D. Wouessidjewe, Physicochemical characterization of $\alpha-, \beta$ - and $\gamma$-cyclodextrins bioesterified with decanoate chains used as building blocks of colloidal nanoparticles, Biomacromolecules 12 (8) (2011) 3031-3038.

[21]L. Choisnard, A. Gèze, J.-L. Putaux, Y.S. Wong, D. Wouessidjewe, Novel nanoparticles of $\beta$-cyclodextrin esters obtained by self-assembling of bio-transesterified $\beta$-cyclodextrins, Biomacromolecules 7 (2006) 515520.

[22]L. Choisnard, A. Gèze, B.G.J. Yaméogo, J.-L. Putaux, D. Wouessidjewe, Miscellaneous nanoaggregates made of CD esters synthesized by an enzymatic pathway, Int. J. Pharm. 344 (2007) 26-32.

[23] A. Gèze, L. Choisnard, J.-L. Putaux, D. Wouessidjewe, Colloidal systems made of biotransesterified cyclodextrins grafted with C10 alkyl chains, Mater. Sci. Eng. C 29 (2009) 458-462.

[24] J.B. Yaméogo, A. Gèze, L. Choisnard, J.-L. Putaux, R. Mazet, C. Passirani, M. Keramidas, J.-L. Coll, N. Lautram, J. Bejaud, R. Semdé, D. Wouessidjewe, Self-assembled biotransesterified cyclodextrins as potential artemisinin nanocarriers. II: In vitro behavior toward the immune system and in vivo biodistribution assessment of unloaded nanoparticles, Eur. J. Pharm. Biopharm. 88 (2014) 683-694.

[25]P. Perret, S. Bacot, A. Gèze, A. Gentil Dit Maurin, M. Debiossat, A. Soubies, V. Blanc-Marquis, L. Choisnard, J. Boutonnat, J.-L. Putaux, C. Lancelon-Pin, L.M. Riou, D. Wouessidjewe, Biodistribution and preliminary toxicity studies of nanoparticles made of biotransesterified $\beta$-cyclodextrins and PEGylated phospholipids, Mater. Sci. Eng. C 85 (2018) 7-17.

[26] K.V. Thrivikraman, R.L. Huot, P.M. Plotsky, Jugular vein catheterization for repeated blood sampling in the unrestrained conscious rat, Brain Res. Protoc.10 (2002) 84-94.

[27]B.A. Avery, K.K. Venkatesh, M.A Avery, Rapid determination of artemisinin and related analogues using high-performance liquid chromatography and an evaporative light scattering detector, J. Chromat. B 730 (1999) 71-80.

[28] M. Ashton, T. Gordi, T.N. Hai, N. Van Huong, N.D. Sy, N.T. Nieu, D.X. Huong, M. Johansson, D. Công, Artemisinin pharmacokinetics in healthy adults after 250, 500 and $1000 \mathrm{mg}$ single oral doses, Biopharm. Drug Dispos. 19 (1998) 245-250.

[29] A.A. Lapkin, A. Walker, N. Sullivan, B. Khambay, B. Mlambo, S. Chemat, Development of HPLC analytical protocols for quantification of artemisinin in biomass and extracts, J. Pharm. Biomed. Anal. 49 (2009) 908-915.

[30] T. Dai, W. Jiang, Z. Guo, Y. Xie, R. Dai, Comparison of in vitro/in vivo blood distribution and pharmacokinetics of artemisinin, artemether and dihydroartemisinin in rats, J. Pharm. Biomed. Anal. 162 (2019) 140-148.

[31] M. Ashton, M. Johansson, A.S. Thorngvist, U.S. Svensson, Quantitative in vivo and ex vivo sex difference in artemisinin metabolism in rats, Xenobiotica 29(2) (1999) 195-204.

[32] B. Isacchi, S. Arrigucci, G. la Marca, M.C. Bergonzi, M.G. Vannucchi, A. Novelli, A.R. Bilia, Conventional and long-circulating liposomes of artemisinin: preparation, characterization, and pharmacokinetic profile in mice., J. Liposome Res. 21(3) (2011) 237-244.

[33] H.K. Manjili, H.M., M.S. Mousavi, E. Attari, H. Danafar, In vitro and in vivo delivery of artemisinin loaded PCL-PEG-PCL micelles and its pharmacokinetic study, Artif. Cells Nanomed. Biotechnol. 46(5) (2018) 926-936. 